\section{Cerebral emboli and depressive symptoms}

\section{in dementia}

\author{
NITIN PUR ANDARE, RICHARD C. OUDE VOSHAAR, JAYNE HARDICRE, \\ JANE BYRNE, CHARLES MCCOLLUM and ALISTAIR BURNS
}

\begin{abstract}
Background The vascular depression hypothesis and our recent findings of increased frequency of spontaneous cerebral emboli in dementia suggest that such emboli may be involved in the causation of depressive symptoms in dementia.
\end{abstract}

\begin{abstract}
Aims To evaluate the association between spontaneous cerebral emboli and depressive symptoms in Alzheimer's disease and vascular dementia.
\end{abstract}

Method In a cohort of 142 patients with dementia (72 with Alzheimer's disease and 70 with vascular dementia), the association between spontaneous cerebral emboli and clinically relevant depressive symptoms was examined using multiple logistic regression analyses.

\section{Results Spontaneous cerebral emboli were significantly more frequent in the patients with clinically relevant depressive symptoms ( 66 v. $37 \%, P=0.03$ ). After adjustment for age, gender, Mini-Mental State Examination score, type of dementia and significant cardiovascular risk factors, the relationship remained significant $(\mathrm{OR}=3.47,95 \% \mathrm{Cl}$ I.I0-10.97).}

\section{Conclusions Spontaneous cerebral emboli are associated with clinically relevant depressive symptoms in} dementia, and further research is needed to explore the nature of this relationship.

Declaration of interest None. Funding detailed in Acknowledgements.
Depression affects up to $85 \%$ of patients with dementia (Wragg \& Jeste, 1989; Weiner et al, 2002), with a negative impact on both patients and caregivers (Black $\&$ Almeida, 2004). The vascular depression hypothesis (Alexopoulos et al, 1997) suggests that cardiovascular risk factors have a causative role in depressive symptoms among older adults, but research on the vascular depression hypothesis in dementia is limited. Recently we detected spontaneous cerebral emboli in the middle cerebral arteries of about $40 \%$ of patients with dementia (Purandare et al, 2006). Such microemboli could be asymptomatic, but are recognised as a risk factor for cerebrovascular accidents and poor neurocognitive outcome in various patient groups (Russell, 2002). We investigated the relationship between cerebral emboli, cardiovascular factors and depressive symptoms in patients with dementia, with the hypothesis that spontaneous cerebral emboli would be independently related to depressive symptoms in dementia.

\section{METHOD}

\section{Patients}

Patients with dementia were drawn from participants in an earlier study investigating the frequencies of spontaneous cerebral emboli in Alzheimer's disease and vascular dementia, and the method has been described in detail by Purandare et al (2006). Briefly, patients were recruited from secondary care old age psychiatry services in Greater Manchester, UK, and satisfied either the National Institute of Neurological and Communicative Disorders and StrokeAlzheimer's Disease and Related Disorders Association (NINCDS-ADRDA) criteria for Alzheimer's disease (McKhann et al, 1984) or the National Institute of Neurological Disorders and Stroke-Association Internationale pour la Recherche et l'Enseignement en Neurosciences (NINDS-
AIREN) criteria for vascular dementia (Roman et al, 1993). The diagnoses were made by an old age psychiatrist (J.B.), who was independent of the research team and was not involved in patient care in most cases. Patients with severe dementia, defined as a score below 10 on the Mini-Mental State Examination (MMSE; Folstein et al, 1975), and those receiving anticoagulant treatment were excluded. The local research ethics committees approved the study, and only patients (and their carers) who gave written informed consent were included.

\section{Depressive symptoms}

Depressive symptoms were assessed at an interview with carers using the Neuropsychiatric Inventory (NPI; Cummings et al, 1994), which rates depressive symptoms and 11 other non-cognitive symptoms in the patient over the previous month. If the screening question is answered positively, the severity is classified into 'mild', 'moderate' or 'severe' (score 1-3) and the frequency into 'less than once a week', 'once a week', 'most days, but not every day' and 'every day' (score 1-4). The NPI symptom score is calculated by multiplying the scores for severity and frequency, which gives a range of $1-12$ if a symptom is present and a score of 0 if a symptom is absent. A score of 4 or more on the depressive symptom sub-scale (mild symptoms occurring every day, or moderate to severe symptoms occurring at least once a week) was used to identify clinically relevant depressive symptoms (Aalten et al, 2005; Holthoff et al, 2005).

\section{Cerebral emboli}

The presence of spontaneous cerebral emboli was investigated independently in the Vascular Studies Unit at South Manchester University Hospital by technologists who did not have access to information about dementia type or details of the patients' symptoms. The methods, including the key technical parameters, have been described previously (Ringelstein et al, 1998; Purandare et al, 2006). Briefly, continuous transcranial Doppler insonation of the middle cerebral arteries via the transtemporal windows for $1 \mathrm{~h}$ was used to detect the emboli. The output was recorded on digital tape for subsequent analysis by a vascular technologist, masked to patient identity. Patients were observed during each session for any movement so that artefacts could be identified. Emboli were defined using international consensus 
criteria, which specify that embolic signals should be transient (lasting less than $300 \mathrm{~ms}$ ), at least $3 \mathrm{~dB}$ higher than the background blood flow signal, unidirectional within the Doppler spectrum, and accompanied by an audible 'snap', 'chirp' or 'moan' (Consensus Committee of the Ninth International Cerebral Hemodynamic Symposium, 1995). Detection of one or more embolic signals constituted a positive finding.

\section{Cardiovascular risk factors}

The severity of carotid disease was imaged using colour duplex ultrasound (Ultramark 9; Advanced Technology Laboratories, Inc., Washington, DC, USA). The peak systolic velocity in the internal carotid arteries was used to calculate the degree of stenosis, using established criteria (Sidhu \& Allen, 1997). Blood pressure was measured manually after a 5 min rest. Pulse pressure, calculated as the difference between the systolic and diastolic blood pressures, is related to arterial stiffness and as such was used as a measure of atherosclerosis (Van Popele et al, 2001). A blood sample was taken to assess lipid profile and apolipoprotein E genotype. Data on the following cardiovascular risk factors were collected at interview with patients and their caregivers, and by reviewing case notes: smoking status (current/former/non); alcohol consumption per week over 14 units (women) or 21 units (men); drug history; and history of myocardial infarction, angina, stroke, transient ischaemic attacks, hypertension, hypercholesterolaemia or diabetes.

\section{Statistical analyses}

We used chi-squared statistics or Fisher's exact test to determine the univariate relationship between the NPI depression scores $(\geqslant 4)$ and the presence of spontaneous cerebral emboli. To control for potential confounders we performed multiple logistic regression analyses entering the dichotomised NPI depression scores as the dependent variable and the presence or absence of emboli as the independent variable. The analysis was repeated initially without controlling for the effect of potential confounding variables, and secondarily by using age, gender, type of dementia and level of cognitive functioning (MMSE score) as independent variables. Thereafter, we also adjusted for all cardiovascular risk factors that were univariately associated with depressive symptoms in our sample at the $15 \%$ level. The level of significance in the final model was set at $P<0.05$ (two-tailed).

\section{RESULTS}

\section{Participants}

Of the original cohort of 170 patients with dementia (Purandare et al, 2006), 28 were excluded because NPI data were not available $(n=25)$ or no reliable transtemporal window for detecting cerebral emboli was found $(n=3)$. The excluded patients did not differ significantly from those included with respect to age, gender, diagnosis or MMSE score. Of the 142 patients included, 72 had Alzheimer's disease (63 probable, 9 possible disease) and 70 had vascular dementia (45 probable, 25 possible). The patients' average age was 76.6 (s.d.=7.0) years and $73(51 \%)$ were women. The mean MMSE score was 21.5 (s.d.=4.8). The Alzheimer's disease and vascular dementia groups were similar with regard to age, gender and MMSE scores.

\section{Depressive symptoms and cerebral emboli}

Spontaneous cerebral emboli were detected in $29(40 \%)$ patients with Alzheimer's disease and $28(40 \%)$ patients with vascular dementia (Pearson $\left.\chi^{2}, P=0.97\right)$. The depression screening question suggested the possibility of depressive symptoms in 62 patients ( $44 \%$ of the sample), of whom 15 $(11 \%)$ patients scored 4 or higher on the depression sub-scale of the NPI: $9(13 \%)$ of the 72 patients with Alzheimer's disease and $6(9 \%)$ of the 70 patients with vascular dementia (Pearson $\chi^{2}, P=0.45$ ). The prevalence of this clinically relevant depression was significantly higher among patients with emboli (Table 1; Pearson $\chi^{2}$, $P=0.027)$.

\section{Multivariate analyses}

The groups with Alzheimer's disease and vascular dementia were combined in the multivariate analyses, as neither the prevalence of emboli nor depressive symptoms differed significantly between the two types of dementia. Logistic regression analysis adjusted for age, gender, diagnosis and cognitive functioning (MMSE score) revealed a significant association between the presence of emboli and clinically relevant depressive symptoms: odds ratio $=3.47$ (95\% CI 1.10 10.97), $P=0.034$. Of the cardiovascular
Table I Prevalence of depressive symptoms based on different cut-off points scored on the Neuropsychiatric Inventory

\begin{tabular}{lcc}
\hline $\begin{array}{l}\text { Cut-off score for } \\
\text { depression }\end{array}$ & $\begin{array}{c}\text { Spontaneous cerebral } \\
\text { emboli }\end{array}$ \\
\cline { 2 - 3 } & $\begin{array}{c}\text { Negative } \\
n / N(\%)\end{array}$ & $\begin{array}{c}\text { Positive } \\
n / N(\%)\end{array}$ \\
\hline Whole sample & & \\
$3 / 4$ & $5 / 85(6)$ & $10 / 57(18)^{*}$ \\
$5 / 6$ & $3 / 85(4)$ & $6 / 57(11)$ \\
Alzheimer's disease & & \\
$3 / 4$ & $4 / 43(9)$ & $5 / 29(17)$ \\
$5 / 6$ & $3 / 43(7)$ & $2 / 29 \quad(7)$ \\
Vascular dementia & & \\
$3 / 4$ & $1 / 42(2)$ & $5 / 28(18)^{*}$ \\
$5 / 6$ & $0 / 42(0)$ & $4 / 28(14)^{*}$ \\
\hline
\end{tabular}

$* P<0.05$.

risk factors, only a history of a myocardial infarction and/or angina was univariately associated with depressive symptoms at the $15 \%$ level (Pearson $\chi^{2}, P=0.147$ ). Including this variable in the model did not change the significance, giving a final odds ratio of 3.47 (95\% CI 1.09-11.07), $P=0.035$. None of the included variables had a significant interaction with type of dementia, showing that diagnosis was not a major factor in explaining the association.

Semi-quantitative sensitivity analyses were performed by entering into our final model each non-significant cardiovascular risk factor and its interaction with type of dementia to check for differential effects in Alzheimer's disease and vascular dementia. None of these variables nor their interaction with type of dementia approached significance or substantially confounded the relationship between clinically relevant depressive symptoms and spontaneous cerebral emboli. Furthermore, correction for cardiovascular risk factors that were previously associated with spontaneous cerebral emboli in healthy people (history of stroke or transient ischaemic attack, antiplatelet drugs and body mass index; see Purandare et al, 2006) did not change the results significantly $(\mathrm{OR}=4.19,95 \% \mathrm{CI}$ 1.18-14.90, $P=0.027)$.

\section{DISCUSSION}

The presence of spontaneous cerebral emboli was significantly associated with the 
occurrence of clinically relevant depressive symptoms in patients with dementia, independent of the level of cognitive functioning, age, gender and type of dementia. In addition, the relationship was unaffected by other cardiovascular risk factors, thereby confirming our hypothesis. Our findings are in line with previous research showing increased prothrombotic states and platelet activation in depression (Musselman et al, 1996). Cerebral emboli might be aetiologically linked to depressive symptoms in dementia by causing vascular damage to the frontostriatal pathways involved in depression or their modulating brain areas. Previous research has shown that patients with dementia and depression have significantly more severe white matter lesions (Barber et al, 1999) and cerebral microinfarcts (Ballard et al, 2000) than dementia patients without depression. The arbitrary distinction between white matter hyperintensities and microinfarction suggests at least partial overlap in aetiological factors, and spontaneous cerebral emboli might be one of these factors. Clark et al (1998), however, did not find a significant relationship between a clinical diagnosis of depression and white matter hyperintensities in 31 patients with Alzheimer's disease, although clinician-rated depression scores were higher in patients with large anterior hyperintensities on magnetic resonance imaging.

The lack of association between cardiovascular risk factors and clinically relevant depressive symptoms in our study may be explained by the low prevalence of both in our sample. On the other hand, a substantial number of studies have found that traditional vascular risk factors such as hypertension and diabetes are not an important cause of depression at a population level (Baldwin, 2005). Cervilla et al (2004), for example, followed up 2584 people with moderate hypertension over 54 months and did not find an association between incident depressive symptoms and cardiovascular risk factors. Both general atherosclerotic disease and atrial fibrillation can generate arterial emboli, and both have been associated with depressive symptoms (Lyness et al, 1998; Tiemeier et al, 2004). In our sample, however, the two measures indicative of atherosclerosis (carotid artery disease and pulse pressure) were related neither to depressive symptoms nor to cerebral emboli (data not shown). Although we did not specifically investigate atrial fibrillation, it is unlikely to be a significant confounder because of the exclusion of patients taking anticoagulant medication.

The assessment of clinically relevant depressive symptoms was based on the Neuropsychiatric Inventory, which is a well-validated and highly reliable questionnaire. Forty-four per cent of our population scored positive on the NPI screening question for depression, which is in line with published findings of $25-55 \%$ of dementia patients scoring positive for depressive symptoms on the NPI (Lyketsos et al, 2000; Mirakhur et al, 2004). The significance of these symptoms, however, can be questioned (Lyketsos et al, 2000; Cummings, 2003; Holthoff et al, 2005), and an NPI cut-off score of $3 / 4$ has been recommended to indicate clinically relevant depressive symptoms for clinical trials in Alzheimer's disease (Schneider et al, 2001). Based on the cut-off point of $3 / 4$, the proportion of dementia patients with depressive symptoms decreases markedly and falls in the same range $(11-25 \%)$ as studies in which depression of clinical significance is assessed according to DSM-IV (American Psychiatric Association, 1994) criteria (Burns et al, 1990; Cummings et al, 1995; Weiner et al, 2002). The fact that our prevalence rate of $11 \%$ is at the lower end suggests that patients with depression might have more often declined participation. This might also explain the relatively low proportion of women in our study. Our observation of the univariate association between cerebral emboli and depressive symptoms becomes too low for reliable multivariate analyses. Depressive symptoms in dementia fluctuate over time and are persistent in only a minority of patients (Ballard et al, 1996; Eustace et al, 2002). Starkstein et al (1997) found that in patients with Alzheimer's disease, minor depressive symptoms were transient, whereas more severe symptoms suggestive of major depression persisted for over a year. Our criteria for clinically relevant depressive symptoms are more suggestive of major depression, and our hypothesis about the underlying mechanism - disruption of frontostriatal circuits owing to vascular damage by spontaneous cerebral emboli may explain their persistence.

Some limitations of our study must be addressed. The cross-sectional assessment of depressive symptoms and emboli hinders causative interpretation. Second, the population studied was a convenience sample from specialist secondary care, limiting generalisation. For example, vascular dementia is a heterogeneous disorder, and we recruited relatively few patients with post-stroke dementia, who are thought to be at particular risk of developing depression. Finally, the assessment of depressive symptoms in our study was rather limited. We did not use a separate scale, such as (for example) the Cornell Scale for Depression in Dementia (Alexopolous et al, 1988), to assess depressive symptoms in more depth, and made no attempt to diagnose clinical depression using established diagnostic criteria such as those of DSM-IV or ICD-10 (World Health Organization, 1992).

Although the vascular depression hypothesis is now established, the pathways between vascular risk and depression remain unknown. To our knowledge, depression in dementia has never been linked with spontaneous cerebral emboli. Our results provide preliminary support for the hypothesis that asymptomatic microembolic events might be involved in causation of clinically relevant depressive symptoms in dementia. Further research is required: first, to confirm our findings in a longitudinal design using standardised diagnostic criteria for depression; and second, to examine whether the relationship is unique to depressive symptoms in dementia or can be generalised to late-onset depression in general.

\section{ACKNOWLEDGEMENTS}

The study was funded by a grant from the Wellcome Trust, UK. We thank S. Holiday, J. Shuttleworth and K. Daly for their help with data collection and data entry.

\section{REFERENCES}

Aalten, P., de Vugt, M. E., Jaspers, N., et al (2005)

The course of neuropsychiatric symptoms in dementia. Part I: findings from the two-year longitudinal Maasbed study. International Journal of Geriatric Psychiatry, 20, 523-530.

Alexopoulos, G. S., Abrams, R. C., Young, R. C., et al (1988) Cornell Scale for Depression in Dementia. Biological Psychiatry, 23, 27I-284.

Alexopoulos, G. S., Meyer, B. S., Young, R. C., et a (1997) 'Vascular depression' hypothesis. Archives of General Psychiatry, 54, 915-922.

American Psychiatric Association (1994) Diagnostic and Statistical Manual of Mental Disorders (4th edn) (DSM-IV). Washington, DC: APA.

Baldwin, R. C. (2005) Is vascular depression a distinct sub-type of depressive disorder? A review of causal evidence. International Journal of Geriatric Psychiatry, 20, I-II. 
Ballard, C. G., Patel, A., Solis, M., et al (1996) A oneyear follow-up study of depression in dementia sufferers. British Journal of Psychiatry, 168, 287-291.

Ballard, C., McKeith, I., O'Brien, J., et al (2000) Neuropathological substrates of dementia and depression in vascular dementia, with a particular focus on cases with small infarct volumes. Dementia and Geriatric Cognitive Disorders, II, 59-65.

Barber, R., Scheltens, P., Gholkar, A., et al (1999) White matter lesions on magnetic resonance imaging in dementia with Lewy bodies, Alzheimer's disease, vascular dementia, and normal aging. Journal of Neurology, Neurosurgery and Psychiatry, 67, 66-72.

Black, W. \& Almeida, O. P. (2004) A systematic review of the association between the behavioral and psychological symptoms of dementia and burden of care. International Psychogeriatrics, 16, 295-315.

Burns, A., Jacoby, R. \& Levy, R. (1990) Psychiatric phenomena in Alzheimer's disease. III: disorders of mood. British Journal of Psychiatry, 157, 81-86.

Cervilla, J., Prince, M. \& Rabe-Hesketh, S. (2004) Vascular disease risk factors as determinants of incident depressive symptoms: a prospective community-based study. Psychological Medicine, 34, 635-641.

Clark, L. M., McDonald, W. M., Welsh-Bohmer, K. A., et al (1998) Magnetic resonance imaging correlates of depression in early- and late-onset Alzheimer's disease. Biological Psychiatry, 44, 592-599.

Consensus Committee of the Ninth International Cerebral Hemodynamic Symposium (1995) Basic identification criteria of Doppler microembolic signals. Stroke, 26, 1123

Cummings, J. L. (2003) The impact of depressive symptoms on patients with Alzheimer disease. Alzheimer Disease and Associated Disorders, 17, 61-62.

Cummings, J. L., Mega, M., Gray, K., et al (1994) The Neuropsychiatric Inventory: comprehensive assessment of psychopathology in dementia. Neurology, 44, 2308-2314.

Cummings, J. L., Ross, W., Absher, J., et al (1995) Depressive symptoms in Alzheimer's disease: assessments and determinants. Alzheimer Disease and Associated Disorders, 9, 87-93.

Eustace, A., Coen, R., Walsh, C., et al (2002) A longitudinal evaluation of behavioural and psychological symptoms of probable Alzheimer's disease. International Journal of Geriatric Psychiatry, 7, 968-973.

Folstein, M. F., Folstein, S. E. \& McHugh, P. R. (1975) Mini-Mental State: a practical method for grading the cognitive state of patients for the clinician. Psychiatry Research, 12, 189-198.

Holthoff, V. A., Beuthien-Baumann, B., Kalbe, E., et al (2005) Regional cerebral embolisation in early Alzheimer's disease with clinically significant apathy or depression. Biological Psychiatry, 57, 412-42I.

NITIN PURANDARE, MRCPsych, University of Manchester, Division of Psychiatry, Education and Research Centre, South Manchester University Hospital, Manchester, UK; RICHARD C. OUDE VOSHAAR, MD, PhD, University of Manchester, Division of Psychiatry, Education and Research Centre, South Manchester University Hospital, Manchester, UK and Department of Psychiatry, Radboud University Nijmegen Medical Centre, Nijmegen, The Netherlands; JAYNE HARDICRE, MSc, Vascular Studies Unit, Academic Surgery Unit, University of Manchester; JANE BYRNE, MRCPsych, University of Manchester, Division of Psychiatry, Education and Research Centre, South Manchester University Hospital, Manchester; CHARLES McCOLLUM, MD, FRCS, Vascular Studies Unit, Academic Surgery Unit, University of Manchester; ALISTAIR BURNS, FRCPsych, University of Manchester, Division of Psychiatry, Education and Research Centre, South Manchester University Hospital, Manchester, UK

Correspondence: Dr N. Purandare, University of Manchester, Division of Psychiatry, Education and Research Centre, 2nd floor,Wythenshawe Hospital, Manchester M23 9LT,UK. Tel: +44 (0) I6I 29I 5887; fax: +44 (0)161 291 5882; email: nitin.purandare@manchester.ac.uk

(First received II August 2005, final revision 3 March 2006, accepted 2 May 2006)

Lee, H. B. \& Lyketsos, C. G. (2003) Depression in Alzheimer's disease: heterogeneity and related issues. Biological Psychiatry, 54, 353-362.

Lyketsos, C. G., Steinberg, M., Tschanz, J.T., et al (2000) Mental and behavioural disturbances in dementia: findings from the Cache County Study on memory in aging. American Journal of Psychiatry, 157, $708-714$

Lyness, J. M., Caine, E. D., Cox, C., et al (1998) Cerebrovascular risk factors and later-life major depression. Testing a small-vessel brain disease model. American Journal of Geriatric Psychiatry, 6, 5-13.

McKhann, G., Drachman, D., Folstein, M., et al (1984) Clinical diagnosis of Alzheimer's disease: report of the NINCDS-ADRDA Work Group under the auspices of Department of Health and Human Services Task Force on Alzheimer's Disease. Neurology, 34, 939-944.

Mirakhur, A., Craig, D., Hart, D. J., et al (2004) Behavioural and psychological syndromes in Alzheimer's disease. International Journal of Geriatric Psychiatry, 19 1035-1039.

Musselman, D. L., Tomer, A., Manatunga, A. C., et a (1996) Exaggerated platelet reactivity in major depression. American Journal of Psychiatry, 153, |3|3-1317.

Purandare, N., Daly, K. J., Hardicre, J., et al (2006) Cerebral emboli as a potential cause of Alzheimer's and vascular dementia: case-control study. BM], 332 III9-II24.

Ringelstein, E. B., Droste, D. W., Babikian, V. L., et a (1998) Consensus on microembolus detection by TCD. International Consensus Group on Microembolus Detection. Stroke, 29, 725-729.

Roman, G. C., Tatemichi, T. K., Erkinjuntti, T., et al (1993) Vascular dementia: diagnostic criteria for research studies. Report of the NINDS-AIREN International Workshop. Neurology, 43, 250-260.

Russell, D. (2002) Cerebral microemboli and cognitive impairment. Journal of the Neurological Sciences, 203204, $211-214$.

Schneider, L. S., Tariot, P. N., Lyketsos, C. G., et a (200I) National Institute of Mental Health Clinical Antipsychotic Trials of Intervention Effectiveness (CATIE): Alzheimer disease trial methodology. American Journal of Geriatric Psychiatry, 9, 346-360.

Sidhu, P. S. \& Allen, P. L. (1997) Ultrasound assessment of internal carotid artery stenosis. Clinical Radiology, 52, 654-658.

Starkstein, S. E., Chemerinski, E., Sabe, L., et al (1997) Prospective longitudinal study of depression and anosognosia in Alzheimer's disease. British Journal of Psychiatry, I7I, 47-52.

Tiemeier, H., van Dijck, W., Hofman, A., et al (2004) Relationship between atherosclerosis and late-life depression: the Rotterdam Study. Archives of General Psychiatry, 6I, 369-376.

Van Popele, N. M., Grobbee, D. E., Bots, M. L., et a (200I) Association between arterial stiffness and atherosclerosis: the Rotterdam study. Stroke, 32 454-460.

Weiner, M. F., Doody, R. S., Sairam, R., et al (2002) Prevalence and incidence of major depressive disorder in Alzheimer's disease: findings from two databases. Dementia and Geriatric Cognitive Disorders, 13, 8-12.

World Health Organization (1992) Tenth Revision of the International Classification of Diseases and Related Health Problems (ICD-10). Geneva: WHO.

Wragg, R. E. \& Jeste, D. V. (1989) Overview of depression and psychosis in Alzheimer's disease. American Journal of Psychiatry, 146, 577-587. 